\title{
Biodegradable metal heart stent: characteristics, material options and preliminary testing method
}

\begin{abstract}
Coronary artery disease is a situation where the arteries are affected by the accumulation and build-up of plaque on their walls. The treatment is by percutaneous coronary intervention (PCT) where the plaque is flattened to the walls and a stent is placed to hold up the arteries at the affected area. However, since the heart stent is a foreign object inserted inside the human body, there are many considerations that come into the picture. The considerations include material selection, design, its interaction with the surroundings, and the ability of the device to carry on its task without failing and without causing more issues which may bring more harm to the patient. The discussion starts with the different evolutions of the heart stent and the issues normally related with each generation of heart stent. The focus will then turn to the characteristics that were deemed important for a third generation heart stent which is the biodegradable heart stent. The three main metal options, i.e. magnesium, zinc and ferum, are also discussed. From the attributes it can be seen that each metal has its own positive and negative aspects. The paper finally ends with a discussion of some methods of characterising the heart stent to understand its reaction with the human body, as a preliminary to how it will perform before the testing escalates to animals and human case studies. The biodegradable heart stent can be the future technology of heart stents that will not give side issues (such as thrombosis), which are usually associated with stents. More research will be needed to solve the issues and produce a workable third generation heart stent
\end{abstract}

Keyword: Biodegradable; Characteristics; Ferum; Heart stent; Magnesium; Zinc 